\title{
Motor Neuron Abnormalities Correlate with Impaired Movement in Zebrafish that Express Mutant Superoxide Dismutase 1
}

\author{
Katherine J. Robinson, ${ }^{1}$ Kristy C. Yuan, Emily K. Don, Alison L. Hogan, ${ }^{2}$ Claire G. Winnick, \\ Madelaine C. Tym, Caitlin W. Lucas, Hamideh Shahheydari, Maxinne Watchon,,3 \\ Ian P. Blair, Julie D. Atkin, Garth A. Nicholson,,4 Nicholas J. Cole,, and Angela S. Laird ${ }^{2}$
}

\begin{abstract}
Amyotrophic lateral sclerosis (ALS) is a fatal neurodegenerative disease characterized by progressive loss of motor neurons. ALS can be modeled in zebrafish (Danio rerio) through the expression of human ALS-causing genes, such as superoxide dismutase 1 (SOD1). Overexpression of mutated human SOD1 protein causes aberrant branching and shortening of spinal motor axons. Despite this, the functional relevance of this axon morphology remains elusive. Our aim was to determine whether this motor axonopathy is correlated with impaired movement in mutant (MT) SOD1-expressing zebrafish. Transgenic zebrafish embryos that express blue fluorescent protein (mTagBFP) in motor neurons were injected with either wild-type (WT) or MT (A4V) human SOD1 messenger ribonucleic acid (mRNA). At 48 hours post-fertilization, larvae movement (distance traveled during behavioral testing) was examined, followed by quantification of motor axon length. Larvae injected with MT SOD1 mRNA had significantly shorter and more aberrantly branched motor axons $(p<0.002)$ and traveled a significantly shorter distance during behavioral testing $(p<0.001)$ when compared with WT SOD1 and noninjected larvae. Furthermore, there was a positive correlation between distance traveled and motor axon length $\left(R^{2}=0.357, p<0.001\right)$. These data represent the first correlative investigation of motor axonopathies and impaired movement in SOD1-expressing zebrafish, confirming functional relevance and validating movement as a disease phenotype for the testing of disease treatments for ALS.
\end{abstract}

Keywords: amyotrophic lateral sclerosis, motor neurons, behavioral testing, chemical screening, motor neuron disease

\section{Introduction}

A MYOTROPHIC LATERAL SCLEROSIS (ALS), also known as motor neuron disease, is a fatal neurodegenerative disease characterized by progressive loss of motor neurons. ${ }^{1}$ Patients with ALS develop muscle atrophy and paralysis, originating in either the limbs or bulbar regions, and usually die within 3-5 years of diagnosis. ${ }^{2}$ While most cases of ALS are considered sporadic, around $10 \%$ of ALS is inherited, known as familial ALS (FALS). ${ }^{1}$ Several ALS-causing gene mutations have been identified, including mutations in the superoxide dismutase 1 (SOD1) gene (20\% of FALS ${ }^{3}$ ), TDP43 gene $\left(<5 \%\right.$ of FALS cases), ${ }^{4,5}$ FUS/TLS gene $(5 \%$ of FALS $\left.^{6,7}\right)$, and repeat expansions within the C9ORF72 gene ( $\sim 40 \%-50 \%$ of FALS). ${ }^{8,9}$

Unfortunately, there are currently no treatments available to patients with ALS that can produce a meaningful increase in patient's life span and quality of life. ${ }^{10}$ Riluzole, an approved treatment for ALS, is only mildly efficacious, increasing survival by an average of 2 months. ${ }^{11}$ To aid

\footnotetext{
${ }^{1}$ Department of Biomedical Sciences, Faculty of Medicine and Health Sciences, Macquarie University, Sydney, Australia.

${ }^{2}$ Department of Biomedical Sciences, Faculty of Medicine and Health Sciences, Centre for Motor Neuron Disease Research, Macquarie University, Sydney, Australia.

${ }^{3}$ Sydney Medical School, University of Sydney, Sydney, Australia.

${ }^{4}$ Concord Clinical School and ANZAC Research Institute, Concord Repatriation Hospital, Concord, Australia.

(C) Katherine J. Robinson et al. 2018; Published by Mary Ann Liebert, Inc. This Open Access article is distributed under the terms of the Creative Commons License (http://creativecommons.org/licenses/by/4.0), which permits unrestricted use, distribution, and reproduction in any medium, provided the original work is properly cited.
} 
discovery and preclinical testing of novel disease treatments, a range of animal models of ALS have been developed and characterized. The most commonly used animal model of ALS is the mutant (MT) human SOD1 (G93A) mouse model. ${ }^{12}$ Additional mouse models of ALS include those based on other SOD1 mutations, ${ }^{13,14}$ as well as mutations in TDP-43, ${ }^{15-17}$ FUS/ $T L S,{ }^{18,19}$ and C9ORF72. ${ }^{20-22}$ SOD1 mouse models have been used in more than 1300 ALS studies, ${ }^{23}$ with over 60 different molecules yielding protective effects. However, only one of these treatments (riluzole) has proven to be beneficial in ALS patients. ${ }^{10}$ Therefore, additional ALS animal models are needed to aid the testing of potential therapeutics.

Small animal models such as zebrafish (Danio rerio) and nonvertebrates such as worms (Caenorhabditis elegans) and flies (Drosophila melanogaster) offer a range of advantages for the study of neurodegenerative diseases, particularly for testing of potential therapeutic candidates. ${ }^{24}$ Zebrafish are a particularly useful tool because large numbers of zebrafish can be housed in a small space, at relatively low cost, and bred rapidly to provide large sample sizes. ${ }^{25}$ Zebrafish also possess many physiological and anatomical similarities to humans, ${ }^{25-27}$ are transparent during development, ${ }^{28}$ and can be genetically modified to express fluorescent proteins. $^{29}$ Finally, zebrafish can absorb compounds added to water, ${ }^{30}$ and they develop movement within 30 hours postfertilization $(\mathrm{hpf})^{31,32}$ providing a simple and rapid readout of therapeutic efficacy. For these reasons, zebrafish have been used extensively in recent years to study neurodevelopmental and neurodegenerative disorders such as Parkinson's disease, Huntington's disease, spinocerebellar ataxia, hereditary spastic paraplegia, ALS, and spinal muscular atrophy. 5,25,33-44

Several of these studies have demonstrated that expression of human disease-causing proteins can result in development of motor axon abnormalities in zebrafish models of movement diseases. $^{25,33,35,40,41,43-45}$ For example, transient overexpression of mutated human SOD1 has been shown to lead to the development of short and aberrantly branched spinal motor axons. ${ }^{35,38,40}$ Interestingly, these effects appear to be specific to motor neurons with no defects detected in other neuronal populations such as Mauthner neurons or Rohon-Beard sensory neurons. ${ }^{35}$ Despite these findings, studies are yet to confirm whether the presence of motor axon abnormalities in zebrafish embryos or larvae is directly correlated with the development of impaired movement in zebrafish. The present study aims to test for a correlation between motor axon abnormalities and impaired movement in a transient overexpression model of SOD1, by measuring both parameters in the same animals. We hypothesize that zebrafish overexpressing mutated human SOD1 will travel a shorter distance in response to a light stimulus and will display aberrantly branched or shortened motor axons. More so, we predict those that have shorter motor neuron axons will swim shorter distances during testing. Investigating whether this correlation is present will confirm the functional relevance of abnormal motor axon morphology and validate the usefulness of transient SOD1 overexpression models for high-throughput drug screening studies.

\section{Materials and Methods}

\section{Experimental animals}

All experiments were performed in compliance with the Animal Ethics Committee and the Biosafety Committee,
Table 1. Pooled Data Relating to the Number of Embryos That Were of Normal Morphology, AbNormal Morphology, or UNDEVEloped/DEAD FOR EACH GROUP

\begin{tabular}{lcccc}
\hline Group & Normal & Abnormal & $\begin{array}{c}\text { Undeveloped/ } \\
\text { dead }\end{array}$ & $\begin{array}{c}\text { Total } \\
\mathrm{N}\end{array}$ \\
\hline Noninjected & 40 & 1 & 51 & 92 \\
WT SOD1 & 44 & 15 & 36 & 95 \\
MT SOD1 & 37 & 8 & 46 & 91 \\
\hline
\end{tabular}

The data were pooled from four separate experiments. Only larvae with normal morphology were studied within the remaining experiments.

WT, wild type; MT, mutant; SOD1, superoxide dismutase 1.

Macquarie University (NSW, Australia) under ARA 2015034 and NLRD 52014007. Adult zebrafish from the Tg(-3.0mnx1:mTagBFP)mq10 (ZFIN ID: ZDB-TGCONSTRCT$160815-5)^{29}$ zebrafish line (which express blue fluorescent protein in motor neurons) were mated and embryos were collected for injection of the human SOD1 messenger ribonucleic acid (mRNA). The data presented here are pooled from four different rounds of the experiment, resulting in a total of 121 embryos collected from five clutches. Each clutch of embryos were divided into one of three groups; embryos injected with MT human SOD1 mRNA, embryos injected with wild-type (WT) human SOD1 mRNA, and noninjected embryos as a control. Any embryos deemed to be developing abnormally, lacking expression of fluorescent proteins or dead, were excluded from the experiment (Table 1).

\section{Preparation of human SOD1 mRNA and microinjection}

Human SOD1 mRNA was generated through the use of a T7 In Vitro Transcription kit (Ambion; Applied Bioscience). First, a human SOD1 plasmid (WT or containing A4V mutation, within pCMV construct, a kind gift from Wim Robberecht) was linearized with $P c i$ I and the deoxyribonucleic acid (DNA) was purified. One microgram of purified DNA was then transcribed through the use of an mMessage Machine T7 In Vitro Transcription kit (Ambion; Applied Bioscience) and purified by a MEGAclear Kit (Ambion; Applied Bioscience) followed by lithium chloride precipitation.

Microinjection of the human mRNA was performed at 1-4 cell stage with a bolus of $1.15 \mathrm{~nL}$ injected into each embryo containing $250 \mathrm{ng} / \mu \mathrm{L}$ of the SOD1 mRNA and $200 \mathrm{ng} / \mu \mathrm{L}$ of mKate 2 mRNA encoding a red fluorescent protein to allow selection of appropriately injected embryos. At $30 \mathrm{hpf}$, the zebrafish embryos were screened for successful injection (expression of the red fluorescent protein) and the embryos were manually dechorionated with surgical forceps. At $48 \mathrm{hpf}$, positive embryos were then distributed into a 96-well plate, with one embryo placed within each well containing $250 \mu \mathrm{L}$ of $\mathrm{E} 3$ medium $(5 \mathrm{nM} \mathrm{NaCl}, 0.17 \mathrm{mM} \mathrm{KCl}, 0.33 \mathrm{mM}$ $\mathrm{CaCl}_{2}$, and $\left.0.33 \mathrm{mM} \mathrm{MgSO}_{4}\right)$. The plate was incubated at $28^{\circ} \mathrm{C}$ for $10 \mathrm{~min}$ before behavioral testing.

\section{Motor behavioral testing}

Motor function testing was performed within a Zebrabox (Viewpoint) automated zebrafish movement recording device with ZebraLab (Viewpoint) software. At $48 \mathrm{hpf}$, the 96- 
well plates, containing the zebrafish larvae, were moved to the Zebrabox and acclimatized to dark conditions for $10 \mathrm{~min}$. A photomotor response test was then performed, involving exposing the animals to a $1-\mathrm{s}, 300 \mathrm{~W}$ flash of light. The light stimulus was repeated three times at 1-min intervals. The total distance traveled by each animal during the 4-min test period was calculated. The photomotor response was selected as the behavioral test for this experiment as it is a nonvisual reflex that results in a motor response. ${ }^{46}$ The photomotor response can be elicited from $30 \mathrm{hpf}$ and can be performed using a 96-well plate, thus is a suitable behavior test for highthroughput analysis.

Tracking of 6 days post-fertilization (dpf) larvae was conducted in 24-well plates within a Zebrabox tracking machine (Viewpoint). The escape response to darkness involved conditions of 6-min light, 4-min dark, and 4-min light. The total distance traveled by each larva within the dark phase was calculated (first light phase was for acclimatization).

\section{Imaging and quantification of motor axon abnormalities}

Following movement testing, larvae were individually anesthetized through addition of $30 \mu \mathrm{L}$ of $3 \mathrm{mg} / \mathrm{mL}$ tricaine (MS222) to each well of the 96-well plate. Larvae were individually wet mounted onto glass slides and imaged using a fluorescent microscope (Leica DMi8 inverted microscope, Wetzlar, Germany). The average axonal length for each larva was determined by measuring the length of the first five ventral projections of the primary motor neuron axons (ventral root) immediately caudal to the yolk sac (a simple landmark and a region where the axons were of similar length). The axon length was measured using ImageJ with the NeuronJ plugin by measuring the length from the cell body to the distal tip of the axon. The morphology of these first five axons was also examined and the number of aberrantly branched motor axons was counted. Experimenters were blinded to experimental groups during behavioral testing and image analysis stages of the experiment.

\section{Manual cell counting}

The number of cell bodies present within a region caudal to the yolk sac spanning five motor axons long was counted using the particle analysis function within ImageJ (NIH). These cell body counts were performed on the same spinal cord images that the axonal length measurements were performed on.

\section{Western blotting}

Protein lysates were prepared from whole zebrafish embryos in RIPA buffer ( $10 \mathrm{mM}$ Tris- $\mathrm{Cl}$ [pH 8.0], $1 \mathrm{mM}$ ethylenediaminetetraacetic acid [EDTA], $0.5 \mathrm{mM}$ ethylene

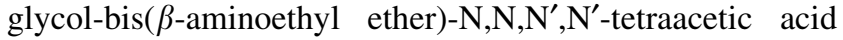
tetrasodium [EGTA], 1\% Triton X-100, 0.1\% sodium deoxycholate, $0.1 \%$ sodium dodecyl sulfate [SDS], $140 \mathrm{mM}$ $\mathrm{NaCl}$ ), by performing hand homogenization using a manual
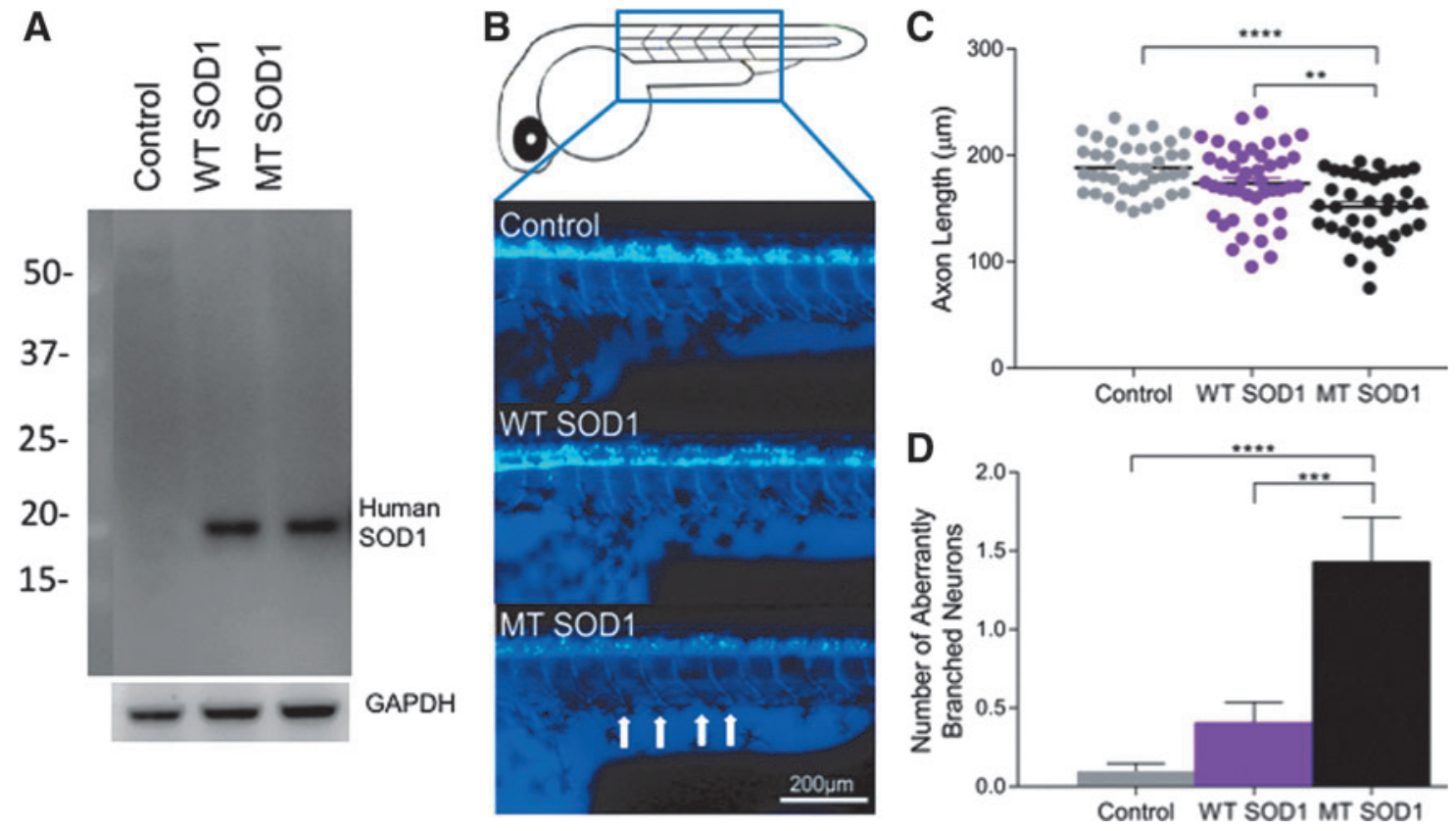

FIG. 1. (A) Expression of human SOD1 protein in zebrafish embryos injected with WT or MT (A4V) human SOD1 was confirmed via Western blot analysis (human SOD1 detected at $\sim 20 \mathrm{kDa}$ ). GADPH was used as a loading control (37 kDa). (B) Representative images of the first five spinal motor neurons after the zebrafish yolk sac are shown for zebrafish larvae at 48 hours postfertilization. Control (noninjected) and larvae that expressed WT human SOD1 displayed long, J-shaped motor axons, while those that expressed MT SOD1 had axons that were shorter in length (arrows). (C) Motor neuron axon length analysis revealed larvae that expressed MT SOD1 had significantly shorter axons than noninjected controls $(* * * * p<0.001)$ or larvae that expressed WT SOD1 $(* * p=0.004)$. There was no statistically significant difference in motor axon length between controls and larvae that expressed WT SOD1. (D) Larvae that expressed MT SOD1 had significantly more aberrantly branched axons per embryo than noninjected controls $(* * * * p<0.001)$ or those expressing WT SOD1 $(* * * p=0.001)$. Each dot represents an individual larva; noninjected: $n=40$; WT SOD1: $n=44$; MT SOD1: $n=37$. WT, wild type; MT, mutant; SOD1, superoxide dismutase 1. 
Dounce homogenizer. Protein concentration was quantified via BCA protein concentration assay (Pierce; Thermo Fisher Scientific) and equal amounts of protein sample $(50 \mu \mathrm{g})$ were loaded for separation via SDS-PAGE. Proteins were transferred from the gel to a polyvinylidene fluoride (PVDF) membrane and blocked with 5\% milk solution. Human SOD1 was probed for using a monoclonal SOD1 antibody (1:5000; Santa Cruz, Catalog \# SC-17767, RRID:AB_628301) and anti-mouse horseradish peroxidase secondary antibody (Promega), followed by ECL chemiluminescence imaging. GAPDH was probed as a loading control using mouse anti-GAPDH (Proteintech; Cat \# 60004-1-lg, 1:10,000).

\section{Statistical analysis}

Data were compared using one-way analysis of variance (ANOVA), followed by Tukey post-hoc analysis using SPSS (version 21.0). The presence of a correlation between the axonal length of each animal with the distance that the animal swam during the photomotor response was measured using a Pearson's correlation test, calculating the relevant $R^{2}$ and $p$-value.

\section{Results}

Expression of the human SOD1 protein (WT or MT [A4V]) was confirmed via Western blotting analysis of protein lysates extracted from injected embryos (Fig. 1A). Measurement of the length of motor axons within the injected zebrafish larvae revealed that expression of human MT SOD1 affected the axonal outgrowth (one-way ANOVA, $p<0.001$ ). Noninjected and WT SOD1 expressing larvae possessed long J-shaped motor axons, while those overexpressing MT SOD1 had axons that were shorter in length (Fig. 1B, C). Tukey post-hoc analysis revealed that larvae injected with MT SOD1 had significantly shorter axons than larvae injected with WT SOD1 (151.53 \pm 5.00 vs. $173.64 \pm 4.56 ; n=37-44$; $p=0.004)$ and noninjected larvae $(188.34 \pm 4.78 ; n=44$; $p<0.001$ ) (Fig. 1C). Larvae injected with MT SOD1 also had significantly more axons with aberrant branching than larvae injected with WT SOD1 (1.432 \pm 0.179 vs. 0.409 \pm 0.164 ; $p<0.001)$ or noninjected larvae $(0.100 \pm 0.172, p<0.001)$ (Fig. 1D). Despite the presence of aberrant motor axons, cell counting did not reveal any neuronal loss present within MT SOD1-expressing compared with WT SOD1 or noninjected larvae $(p=0.7381$, Supplementary Fig. S1).

Examination of the movement of the zebrafish to a flash of light (photomotor response) also revealed an effect of human SOD1 expression, as indicated by images of the trajectory of movement of zebrafish in response to flash of light (Fig. 2A). One-way ANOVA comparison revealed that there was a significant group effect on the movement of animals (Fig. 2B, $p=0.004$ ) and Tukey post-hoc analysis revealed that MT SOD1 larvae traveled a significantly shorter distance than those expressing WT human SOD1 (12.11 \pm 3.04 vs. $25.50 \pm 2.79 ; n=37-44 ; p=0.004)$, and noninjected larvae (23.39 $\pm 2.92 ; n=40 ; p=0.023)$. No difference was found between the movement of the animals during an escape response to darkness test at $6 \mathrm{dpf}$, at which point the human SOD1 protein was no longer expressed ( $p=0.2152$; Supplementary Fig. S2).

Examination of the axon length and distance traveled, both measured within the same individual animals, revealed that
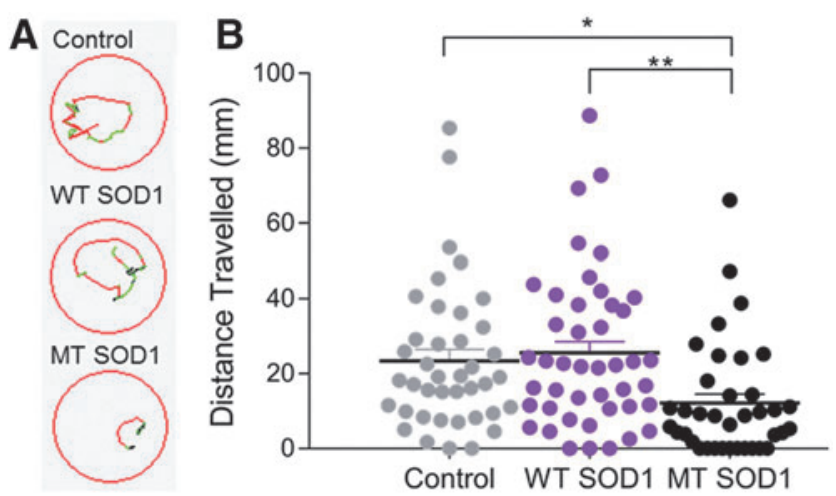

FIG. 2. (A) Representative images displaying the trajectory of movement of individual larvae during a photomotor response test within a 96 multiwell plate (red lines indicate fast movement, green lines indicate slow movement, and black lines indicate inactivity). (B) Larvae injected with MT SOD1 traveled a significantly shorter distance during the photomotor response test compared with noninjected controls $\left({ }^{*} p=0.023\right)$ and those that expressed WT SOD1 $(* * p=0.004)$. There was no statistically significant difference in the distance traveled by WT SOD1 and control larvae. Each dot represents an individual larva; noninjected: $n=40$; WT SOD1: $n=44$; MT SOD1: $n=37$.

there was a positive correlation between distance traveled and axon length (Fig. $3, R^{2}=0.359$ ), which was statistically significant $(p<0.001)$.

\section{Discussion}

In this study, we present the first quantitative correlative report of motor axonopathy and impaired movement in zebrafish larvae overexpressing human MT (A4V) SOD1. We observed a significant axonopathy (shorter and aberrantly branched spinal motor neuron axons), similar to that described in previous studies examining transient overexpression of MT SOD1 in zebrafish. ${ }^{35,38,40} \mathrm{We}$ found that the shorter motor axon length was correlated with an impaired movement phenotype in the same animals. While other studies have reported movement phenotypes in MT SOD1-expressing zebrafish, ${ }^{37,38,40,47}$ we have used the

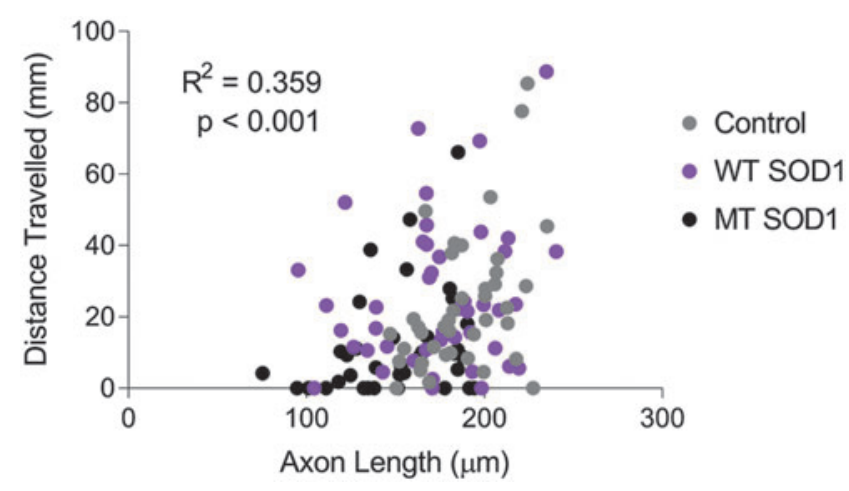

FIG. 3. Decreased axonal length of MT SOD1 expressing spinal motor axons is moderately correlated with impaired movement in zebrafish expressing mutant SOD1 $\left(R^{2}=0.359\right.$, $p<0.001)$. Each dot represents an individual larva; noninjected: $n=40$; WT SOD1: $n=44$; MT SOD1: $n=37$. 
photomotor response assay, which is a useful movement assay for drug screening studies because the behavioral responses can be detected as early as $30 \mathrm{hpf}$ in an automated manner, reducing experimenter labor (e.g., no need to manually induce movement of each animal). Furthermore, the photomotor response consistently delivers the same stimulus to all examined larvae, as opposed to touch-evoked escape responses that can vary in the intensity, timing, and location of applied stimuli. The test does not rely on the visual system, instead a motor response is elicited following activation of photoreceptors in the hindbrain of the zebrafish. ${ }^{46}$ In this study, we chose to perform the photomotor response assay and imaging for axonopathies both at $48 \mathrm{hpf}$, as we found that this was the most suitable time point for detecting larvae in the tracking device, due to pigment development, while still allowing clear imaging of the developing motor axons. While previous studies have measured motor axons at earlier time points (e.g., $30 \mathrm{hpf}^{35}$ ), we found that the enlarged yolk sac at that age complicated imaging of motor axons and we were unable to perform automated movement tracking at that age. While we did not detect any decrease in motor neuron numbers produced by expression of MT SOD1, findings by Sakowski et al. ${ }^{40}$ suggest that motor neuron loss may occur at a later stage.

In future studies, it would be valuable to confirm the finding of axonal outgrowth defects, and correlated movement impairment, in transgenic zebrafish larvae that stably express MT SOD1, rather than zebrafish transiently overexpressing human SOD1. Use of a stable transgenic SOD1 zebrafish line, such as those reported previously, ${ }^{37,40,42,47}$ would reduce labor and experimental variation associated with mRNA injection. It would also be useful to explore whether the phenotypes are present when human SOD1 protein is expressed at closer to endogenous levels, rather than through use of an overexpression model. Transient overexpression of human mRNA, such as MT and WT SOD1, can cause toxicity, as evidenced by heightened mortality and abnormal morphology in injected animals in this experiment. Such abnormal development could contribute to the axonal and behavioral changes we observed and may explain why motor axonopathies have not been previously reported to occur in transgenic SOD1 zebrafish models. $^{37,40,42}$ To prevent developmental abnormalities from occurring, the amount of human SOD1 mRNA injected, and human SOD1 protein expressed, was kept at a lower level than in a previously reported transient SOD1 zebrafish model. ${ }^{35}$ Furthermore, we were careful to only study animals with normal gross morphology (body shape and length), to limit the effect of developmental delay.

This study only examined one SOD1 mutation (A4V). Further studies of the effect of other SOD1 mutations and other MT ALS genes on zebrafish larva movement would be useful for establishing drug screening studies. We previously reported correlation of axonopathy and impaired movement in a zebrafish model of ALS linked to MT cyclin F. ${ }^{43}$ This suggests that this could be a common occurrence in zebrafish models of ALS.

This is the first time that decreased axonal length in embryos overexpressing SOD1 has been found to be quantitatively correlated with a functional impairment. Our findings validate previous investigations that have examined axonopathy as the sole measure of neurodegeneration, including studies that screened for genetic modifiers. ${ }^{48} \mathrm{We}$ confirm that the motor neuron axonopathy is a relevant disease readout for such studies, but we also suggest that measuring zebrafish movement may provide another useful and potentially more high-throughput readout for such studies, with a clear relevance to movement disorders such as ALS.

We conclude that zebrafish that transiently express MT human SOD1 protein develop abnormal motor axon morphology that is correlated with impaired movement. This indicates that behavioral measures, such as movement, will be useful when investigating ALS in zebrafish models of disease, including in studies to discover and test potential therapeutic agents.

\section{Acknowledgments}

The authors thank the staff of the zebrafish facility at the Faculty of Medicine and Health Sciences, Macquarie University, including Rola Bazzi and Jason Martin-Powell. They also thank the laboratory of Prof. Wim Robberecht, especially Mieke Timmers, for providing the human SOD1 DNA construct. K.J.R. was supported by a scholarship from the Office of Pro-Vice-Chancellor, Learning and Teaching, Macquarie University. The research was supported by the Snow Foundation of Australia, Macquarie University Safety Net Scheme, NHMRC Dementia Teams Grant 1095215, NHMRC Project Grant 1069235, 1146750, and Motor Neuron Disease Research Institute of Australia (MNDRIA GIA 1827).

\section{Availability of Data and Material}

Data sharing is not applicable to this article as no data sets were generated or analyzed during the current study.

\section{Authors' Contributions}

K.J.R. performed the zebrafish studies and data analysis and interpretation. K.C.Y. performed the Western blot experiments. E.K.D., A.L.H., C.G.W., M.C.T., C.W.L., H.S., and M.W. assisted with the zebrafish studies. G.A.N., I.P.B., J.D.A., and N.J.C. assisted with experimental design. A.S.L. designed the experiments and performed data analysis and interpretation. All authors assisted with article preparation and approved the final article.

\section{Disclosure Statement}

No competing financial interests exist.

\section{References}

1. Taylor JP, Brown RH, Jr., Cleveland DW. Decoding ALS: From genes to mechanism. Nature 2016;539:197.

2. Kiernan M, Vucic S, Cheah B, Turner M, Fisen A, Hardiman O, et al. Amytrophic lateral sclerosis. Lancet 2011;377:942-955.

3. Rosen DR, Siddique T, Patterson D, Figlewicz DA, Sapp P, Hentati $\mathrm{A}$, et al. Mutations in $\mathrm{Cu} / \mathrm{Zn}$ superoxide dismutase gene are associated with familial amyotrophic lateral sclerosis. Nature 1993;362:59.

4. Sreedharan J, Blair IP, Tripathi VB, Hu X, Vance C, Rogelj $\mathrm{B}$, et al. TDP-43 mutations in familial and sporadic amyotrophic lateral sclerosis. Science 2008;319:1668-1672.

5. Kabashi E, Lin L, Tradewell ML, Dion PA, Bercier V, Bourgouin P, et al. Gain and loss of function of ALSrelated mutations of TARDBP (TDP-43) cause motor deficits in vivo. Hum Mol Genet 2010;19:671-683. 
6. Kwiatkowski TJ, Bosco DA, LeClerc AL, Tamrazian E, Vanderburg CR, Russ C, et al. Mutations in the FUS/TLS gene on chromosome 16 cause familial amyotrophic lateral sclerosis. Science 2009;323:1205-1208.

7. Vance C, Rogelj B, Hortobágyi T, De Vos KJ, Nishimura AL, Sreedharan J, et al. Mutations in FUS, an RNA processing protein, cause familial amyotrophic lateral sclerosis type 6. Science 2009;323:1208-1211.

8. DeJesus-Hernandez M, Mackenzie IR, Boeve BF, Boxer AL, Baker M, Rutherford NJ, et al. Expanded GGGGCC hexanucleotide repeat in noncoding region of C9ORF72 causes chromosome 9p-linked FTD and ALS. Neuron 2011;72:245-256.

9. Renton AE, Majounie E, Waite A, Simon-Sanchez J, Rollinson S, Gibbs JR, et al. A hexanucleotide repeat expansion in C9ORF72 is the cause of chromosome 9p21linked ALS-FTD. Neuron 2011;72:257-268.

10. Petrov D, Mansfield C, Moussy A, Hermine O. ALS clinical trials review: 20 Years of failure. Are we any closer to registering a new treatment? Front Aging Neurosci 2017;9:68.

11. Miller R, Mitchell J, Lyon M, Moore D. Riluzole for amyotrophic lateral sclerosis (ALS)/motor neuron disease (MND). Amyotroph Lateral Scler Other Motor Neuron Disord 2003;4:191-206.

12. Gurney M, Pu H, Chiu A, Dal Canto M, Polchow C, Alexander D, et al. Motor neuron degeneration in mice that express a human $\mathrm{Cu}, \mathrm{Zn}$ superoxide dismutase mutation. Science 1994;264:1772-1775.

13. Jonsson P, Graffmo K, Brännström T, Nilsson P, Andersen $\mathrm{P}$, Marklund S. Motor neuron disease in mice expressing the wild type-like D90A mutant superoxide dismutase-1. J Neuropathol Exp Neurol 2006;65:1126-1136.

14. Filali M, Lalonde R, Rivest S. Sensorimotor and cognitive functions in a SOD1(G37R) transgenic mouse model of amyotrophic lateral sclerosis. Behav Brain Res 2011;225: 215-221.

15. Wegorzewska I, Bell S, Carins N, Miller T, Baloh R. TDP43 mutant transgenic mice develop features of ALS and frontotemporal lobar degeneration. Proc Natl Acad Sci U S A 2009;106:18809-18814.

16. Xu Y, Zhang Y, Lin W, Cao X, Stetler C, Dickson D, et al. Expression of mutant TDP-43 induces neuronal dysfunction in transgenic mice. Mol Neurodegener 2011;6:73.

17. Arnold ES, Ling S-C, Huelga SC, Lagier-Tourenne C, Polymenidou M, Ditsworth D, et al. ALS-linked TDP-43 mutations produce aberrant RNA splicing and adult-onset motor neuron disease without aggregation or loss of nuclear TDP-43. Proc Natl Acad Sci U S A 2013;110:E736-E745.

18. Mitchell J, McGoldrick P, Vance C, Hortobagyi T, Sreedharan J, Rogeli B, et al. Overexpression of human wildtype FUS causes progressive motor neuron degeneration in an age- and dose-dependent fashion. Acta Neuropathol 2013; 125:273-288.

19. Sephton C, Tang A, Kulkarni A, West J, Brooks M, Stubblefield J, et al. Activity-dependent FUS dysregulation disrupts synaptic homeostasis. Proc Natl Acad Sci U S A 2014;111:E4769-E4778.

20. Chew J, Gendron T, Prudencio M, Sasaguri H, Zhang Y, Castanedes-Casey $\mathrm{M}$, et al. C9ORF72 repeat expansions in mice cause TDP-43 pathology, neuronal loss, and behavioral deficits. Science 2015;348:1151-1154.

21. Peters OM, Cabrera GT, Tran H, Gendron TF, McKeon JE, Metterville J, et al. Expression of human C9ORF72 hexanucleotide expansion reproduces RNA foci and dipeptide repeat proteins but not neurodegeneration in BAC transgenic mice. Neuron 2015;88:902-909.

22. O'Rourke J, Bogdanik L, Muhammad A, Gendron T, Kim $\mathrm{K}$, Austin A, et al. C9orf72 BAC transgenic mice display typical pathologic features of ALS/FTD. Neuron 2015;88: 892-901.

23. Kim RB, Irvin CW, Tilva KR, Mitchell CS. State of the field: An informatics-based systematic review of the SOD1-G93A amyotrophic lateral sclerosis transgenic mouse model. Amytroph Lateral Scler Frontotemporal Degener 2016;17:1-14.

24. Giacomotto J, Segalat L. High-throughput screening and small animal models, where are we? Br J Pharmacol 2010; 160:204-216.

25. Kabashi E, Champagne N, Brustein E, Drapeau P. In the swim of things: Recent insights to neurogenetic disorders in zebrafish. Trends Genet 2010;26:373-381.

26. Sager JJ, Bai Q, Burton EA. Transgenic zebrafish models of neurodegenerative diseases. Brain Struct Funct 2010;214: 285-302.

27. Schmid B, Haass C. Genomic editing opens new avenues for zebrafish as a model for neurodegeneration. J Neurochem $2013 ; 127$.

28. Rinkwitz S, Mourrain P, Becker T. Zebrafish: An integrative system for neurogenomics and neurosciences. Prog Neurobiol 2011;93:231-243.

29. Don EK, Formella I, Badrock AP, Hall TE, Morsch M, Hortle E, et al. A Tol2 gateway-compatible toolbox for the study of the nervous system and neurodegenerative disease. Zebrafish 2017;14:69-72.

30. Peterson R, Link B, Dowling J, Schreiber S. Small molecule developmental screens reveal the logic and timing of vertebrate development. Proc Natl Acad Sci U S A 2000; 97:12965-12969.

31. Drapeau P, Saint-Amant L, Buss R, Chong M, McDearmid J, Brunstein E. Development of the locomotor network in zebrafish. Prog Neurobiol 2002;68:85-111.

32. Kokel D, Bryan J, Laggner C, White R, Cheung C, Mateus $\mathrm{R}$, et al. Rapid behavior-based identification of neuroactive small molecules in the zebrafish. Nat Chem Biol 2010;6: 231-237.

33. McWhorter M, Monani U, Burghes B, Beattie C. Knockdown of the survival motor neuron $(\mathrm{Smn})$ protein in zebrafish causes defects in motor axon outgrowth and pathfinding. J Cell Biol 2003;162:919-931.

34. Bai Q, Garver J, Hukriede N, Burton EA. Generation of a transgenic zebrafish model of Tauopathy using a novel promoter element derived from the zebrafish eno 2 gene. Nucleic Acids Res 2007;35:6501-6516.

35. Lemmens R, Van Hoecke A, Hersmus N, Geelen V, D'Hollander I, Thijs V, et al. Overexpression of mutant superoxide dismutase 1 causes a motor axonopathy in the zebrafish. Hum Mol Genet 2007;16:2359-2365.

36. Flinn L, Mortiboys H, Volkmann K, Koster R, Ingham P, Bandmann O. Complex I deficiency and dopaminergic neuronal cell loss in parkin-deficient zebrafish (Danio rerio). Brain 2009;132:1613-1623.

37. Ramesh T, Lyon A, Pineda R, Wang C, Janssen P, Canan $\mathrm{B}$, et al. A genetic model of amyotrophic lateral sclerosis in zebrafish displays phenotypic hallmarks of motoneuron disease. Dis Model Mech 2010;3:652-662.

38. Kabashi E, Bercier V, Lissouba A, Liao M, Brunstein E, Rouleau GA, et al. FUS and TARDBP but not SOD1 interact in genetic models of amyotrophic lateral sclerosis. PLoS Genet 2011;7:e1002214. 
39. Laird AS, Robberecht W. Modelling neurodegenerative diseases in zebrafish embryos. Methods Mol Biol 2011;793: 167-183.

40. Sakowski S, Lunn J, Busta A, Oh S, Zamora-Berridi G, Palmer M, et al. Neuromuscular effects of G93A-SOD1 expression in zebrafish. Mol Neurodegener 2012;7:34.

41. Laird AS, Mackovski N, Rinkwitz S, Becker T, Giacomotto J. Tissue-specific models of spinal muscular atrophy confirm a critical role of SMN in motor neurons from embryonic to adult stages. Hum Mol Genet 2016;25:1728-1738.

42. McGown A, Shaw D, Ramesh T. ZNStress: A highthroughput drug screening protocol for identification of compounds modulating neuronal stress in the transgenic mutant sod1G93R zebrafish model of amyotrophic lateral sclerosis. Mol Neurodegener 2016;11:56.

43. Hogan A, Don EK, Rayner SL, Lee A, Laird AS, Watchon M, et al. Expression of ALS/FTD-linked muntant CCNF in zebrafish leads to increased cell death in the spinal cord and an aberrant motor phenotype. Hum Mol Genet 2017;26:2616-2626.

44. Watchon M, Yuan K, Mackovski N, Svahn A, Cole N, Goldsbury $\mathrm{C}$, et al. Calpain inhibition is protective in Machado-Joseph disease zebrafish due to induction of autophagy. J Neurosci 2017;37:7782-7794.

45. Swinnen B, Bento-Abreu A, Gendron TF, Boeynaems S, Bogaert E, Nuyts R, et al. A zebrafish model for C9orf72 ALS reveals RNA toxicity as a pathogenic mechanism. Acta Neuropathol 2018;135:427-433.
46. Kokel D, Dunn TW, Ahrens MB, Alshut R, Cheung CY, Saint-Amant L, et al. Identification of nonvisual photomotor response cells in the vertebrate hindbrain. J Neurosci 2013;33:3834-3843.

47. Da Costa MMJ, Allen CE, Higginbottom A, Ramesh T, Shaw PJ, McDermott CJ. A new zebrafish model produced by TILLING of SOD1-related amyotrophic lateral sclerosis replicates key features of the disease and represents a tool for in vivo therapeutic screening. Dis Model Mech 2014;7: 73-81.

48. Van Hoecke A, Schoonaert L, Lemmens R, Timmers M, Staats KA, Laird AS, et al. EPHA4 is a disease modifier of amyotrophic lateral sclerosis in animal models and in humans. Nat Med 2012;18:1418.

Address correspondence to: Angela S. Laird, PhD Centre for Motor Neuron Disease Research Department of Biomedical Sciences Faculty of Medicine and Health Sciences Macquarie University Sydney, NSW 2109 Australia

E-mail: angela.laird@mq.edu.au 